\title{
Integrated Simulations of H-mode Operation in ITER including Core Fuelling, Divertor Detachment and ELM Control
}

\author{
A.R. Polevoi ${ }^{1}$, A. Loarte ${ }^{1}$, R. Dux ${ }^{2}$, T. Eich $^{2}$, E. Fable ${ }^{2}$, D. Coster ${ }^{2}$, \\ S. Maruyama ${ }^{1}$, S.Yu. Medvedev ${ }^{3}$, F. Köchl' ${ }^{4}$, V.E. Zhogolev ${ }^{5}$ \\ ${ }^{1}$ ITER Organization, Route de Vinon-sur-Verdon, 13067 St Paul Lez Durance, France \\ ${ }^{2}$ Max-Planck Inst. für Plasmaphysik, Boltzmanstraße 2, D-85748 Garching, Germany \\ ${ }^{3}$ Keldysh Institute of Applied Mathematics, Miusskaya 4, 125047 Moscow, Russia \\ ${ }^{4}$ Atominstitut, Technische Universität Wien, Stadionallee 2, 1020 Vienna, Austria \\ ${ }^{5}$ NRC "Kurchatov Institute", Kurchatov sq. 1, 123098 Moscow, Russia
}

E-mail: Alexei.Polevoi@iter.org

\begin{abstract}
ELM mitigation to avoid melting of the tungsten (W) divertor is one of the main factors affecting plasma fuelling and detachment control at full current for high Q operation in ITER. Here we derive the ITER operational space, where ELM mitigation to avoid melting of the $\mathrm{W}$ divertor monoblocks top surface is not required and appropriate control of $\mathrm{W}$ sources and radiation in the main plasma can be ensured through ELM control by pellet pacing. We apply the experimental scaling that relates the maximum ELM energy density deposited at the divertor with the pedestal parameters and this eliminates the uncertainty related with the ELM wetted area for energy deposition at the divertor and enables the definition of the ITER operating space through global plasma parameters. Our evaluation is thus based on this empirical scaling for ELM power loads together with the scaling for the pedestal pressure limit based on predictions from stability codes. In particular, our analysis has revealed that for the pedestal pressure predicted by the EPED1+SOLPS scaling, ELM mitigation to avoid melting of the $\mathrm{W}$ divertor monoblocks top surface may not be required for $2.65 \mathrm{~T}$ H-modes with normalized pedestal densities (to the Greenwald limit) larger than 0.5 to a level of current of 6.5-7.5 MA, which depends on assumptions on the divertor power flux during ELMs and between ELMs that expand the range of experimental uncertainties. The pellet and gas fuelling requirements compatible with control of plasma detachment, core plasma tungsten accumulation and H-mode operation (including post-ELM W transient radiation) have been assessed by $1.5 \mathrm{D}$ transport simulations for a range of assumptions regarding W redeposition at the divertor including the most conservative assumption of zero prompt re-deposition. With such conservative assumptions, the post-ELM W transient radiation imposes a very stringent limit on ELM energy losses and the associated minimum required ELM frequency. Depending on $\mathrm{W}$ transport assumptions during the ELM, a maximum ELM frequency is also identified above which core tungsten accumulation takes place.
\end{abstract}

\section{Introduction}

Integrated simulations of ITER H-mode plasmas including gas and pellet fuelling for core and edge density/divertor power load control and pellet pacing for ELM control have been carried out [1]. In these studies it was shown that the flexibility of the fuelling systems is appropriate to achieve high Q operation in ITER [1]. These simulations also showed that ELM control to ensure appropriate divertor target erosion lifetime is one of the main factors affecting plasma fuelling and detachment control at full current high Q operation in ITER. At lower plasma currents, the power fluxes during ELMs at the ITER divertor are not expected to cause large scale $\mathrm{W}$ divertor melting [2]. For these lower current levels the requirements for the control of ELMs, such as through frequency control by pellet pacing, are determined by the control of the tungsten concentration in the core plasma required to keep plasma in the $\mathrm{H}$-mode regime $[2,3]$. In the present work we extend the analysis in $[2,3]$ by performing integrated simulations of ITER plasmas including the requirements for core and edge plasma fuelling to ensure appropriate fusion performance and control of divertor power loads together with requirements for ELM control by pellet pacing to avoid melting of the W divertor monoblocks top surface (MTS) and acceptable core W plasma levels. To determine the ELM control requirements to avoid melting of the $\mathrm{W}$ divertor MTS, we have evaluated first the H-mode operational space for which ELM control is not required to avoid such melting. This has been done by applying the empirical scaling for the ELM power flux in [4], 
where the peak ELM energy density at the divertor $(\varepsilon)$ is found to be proportional to the pedestal plasma pressure with $\varepsilon \sim K p_{p e d}$, where $K=1-4.5$ covers the range of experimental data and inner-outer divertor ELM power deposition asymmetries, and the expected pedestal plasma parameters in ITER are evaluated from MHD stability predictions [5]. This approach is based on global plasma parameters and provides a refined evaluation with respect to the approach in [2], which considered the area over which ELMs deposit their energy at the divertor as a variable parameter. The estimate of the H-mode operational space with acceptable ELMs is described in section 2. We note here that in the present study we have incorporated the effects of the optimized shape of the ITER divertor target and of the W monoblocks on the acceptable ELM loads to avoid W MTS melting and those related to the divertor power fluxes during ELMs from the detailed evaluation in [6]. We have, however, not analyzed the issues related to the melting of the edges of divertor monoblocks due to the gaps between them; for details on this topic and on the issues related to the optimization of the ITER divertor geometry the readers is referred to [7].

As discussed in [2], in the range of plasma parameters where uncontrolled natural spontaneous ELMs do not lead to large scale W divertor melting, the limitations on the ELM size and frequency are determined by the requirements of the control of plasma contamination by tungsten caused by ELMs. In section 3 we derive scalings for tungsten influx to the plasma core in ITER as a function of the ELM energy loss size from combined simulations by ASTRA, STRAHL, and SOLPS codes described in [3, 8]. Using these scalings we estimate the tungsten influx from the ELMs in ITER H-modes for a range of plasma conditions by applying the continuous ELM and pellet models [1]. In such time averaged simulations neoclassical effects cause the efficient screening of tungsten in the pedestal [3]. Including the time-varying W source during ELMs and the mechanisms for $\mathrm{W}$ outflux and influx during them is, however, expected to lead to more stringent limits on ELM energy losses on the basis of previous studies [3]. Therefore, simulations including the effect of the time-varying $\mathrm{W}$ influx during the ELM as well as the effects of pellet pacing on the fuelling efficiency of high field side pellets are performed to refine the above estimates with more realistic modelling assumptions and with a more up to date design of the ITER fuelling systems. In section 4, we describe the impact of discrete pellets and ELM modelling assumptions on the pellet injection requirements to achieve the required level of ELM control. Finally, in section 5 we discuss the results of the modelling presented in the paper and reach conclusions.

Throughout this paper we use the following units: seconds for time, $t, \Delta t$, MW for the input and radiated powers $P$, MJ for plasma energy and ELM energy losses $W, \Delta W, \mathrm{~m}, \mathrm{~m}^{2}$ and $\mathrm{m}^{3}$ for size $a, R$, area $S$ and volume $V$, respectively, MA for the plasma current $I_{p}, \mathrm{~T}$ for the toroidal magnetic field $B, 10^{19} \mathrm{~m}^{-3}$ for the plasma density $n, \mathrm{keV}$ for the electron and ion temperatures $T, \mathrm{kPa}$ for the plasma pressure $p, \mathrm{~Hz}$ for the frequencies of ELM and injected pellets, $f$, and $\mathrm{MJm}^{-2}$ for energy density, $\varepsilon$. Thus in the rest of the paper the values for the quantities above are given in these units and we omit, when possible, the units in the formulas below. Note that in our consideration we assume $T_{e, p e d}=T_{i, p e d}=T_{p e d}$.

Table 1. Variables and units

\begin{tabular}{|l|lllllllllllll|}
\hline Name & $t, \Delta t$ & $P$ & $W, \Delta W$ & $a, R$ & $S$ & $V$ & $I_{p}$ & $B$ & $T$ & $n$ & $p$ & $f$ & $\varepsilon$ \\
\hline Units & $\mathrm{s}$ & $\mathrm{MW}$ & $\mathrm{MJ}$ & $\mathrm{m}$ & $\mathrm{m}^{2}$ & $\mathrm{~m}^{3}$ & $\mathrm{MA}$ & $\mathrm{T}$ & $\mathrm{keV}$ & $10^{19} \mathrm{~m}^{-3}$ & $\mathrm{kPa}$ & $\mathrm{Hz}$ & $\mathrm{MJm}^{-2}$ \\
\hline
\end{tabular}

\section{Operation space with tolerable ELMs}


As mentioned above, in this study, we consider that the maximum energy density for controlled ELMs in ITER is limited by melting of the divertor monoblock top surface MTS, $\varepsilon_{\max }$ :

$$
\varepsilon_{\|}=\sin \alpha\left(t_{E L M} / t_{E L M, r e f}\right)^{-0.5} \leq \varepsilon_{\max }
$$

where $\varepsilon_{\max }$ is the maximum ELM energy density in ITER that avoids divertor MTS melting for a reference ELM power deposition time, $t_{E L M, r e f}, \varepsilon_{\|}$is the maximum energy density during the ELM along the field line, $\alpha$ is the angle of inclination of the magnetic field line onto the divertor target surface and $t_{E L M} / t_{E L M, r e f}$ is the normalised time of the duration of the ELM power on the divertor surface.

Our analysis of the operational space with ELMs that avoid W divertor MTS melting is based on the following assumptions:

- The reference plasma conditions are given by the ITER $Q=1015$ MA scenario with a pedestal temperature of $T_{\text {ref }}=4.7 \mathrm{keV}$ and a pedestal electron density $n_{\text {ped }} / n_{G W}=0.70$, where $n_{G W}=10 I_{p} / \pi a^{2}$ is the Greenwald limit, $a=2 \mathrm{~m}$. In the following we denote $g \equiv n_{\text {ped }} / n_{G W}$ as the normalized pedestal density to the Greenwald limit.

- For these plasma conditions we assume that uncontrolled ELMs will have an ELM power rise time of $250 \mu \mathrm{s}$ and fall-down time of $500 \mu \mathrm{s}[2,9]$ and that for these plasmas the peak divertor power flux between ELMs is $q_{p k}=10 \mathrm{MWm}^{-2}$. For a $5-10 \mathrm{~mm}$ thick $\mathrm{W}$ monoblock the surface temperature between ELMs in these conditions is $T_{s}=1500^{\circ} \mathrm{C}$ and the corresponding toroidally averaged value of $\varepsilon_{\max } \approx 0.6 \mathrm{MJm}^{-2}$; this value already takes into account the toroidal inclination of the divertor monoblocks to avoid exposure of their poloidal gaps $[6,9]$. This MTS melting energy density threshold corresponds to an ELM parallel energy density of $10.7 \mathrm{MJm}^{-2}$ for the inner divertor with $\alpha_{i n}=3.2^{\circ}\left(3 / q_{95}\right) \approx 0.0197$ $I_{p} / B$ [rad], where $3.2^{\circ}\left(3 / q_{95}\right)$ is the field line incidence angle, and $q_{95}=8.49 B / I_{p}$ is the edge safety factor in ITER for the reference plasma shape used in this modelling. The corresponding numbers for the outer divertor are $12.7 \mathrm{MJm}^{-2}$ for the parallel energy density with $\alpha_{\text {out }}=2.7^{\circ}\left(3 / q_{95}\right)$. Above these values the $\mathrm{W}$ monoblock top surface would melt when $q_{p k}=10 \mathrm{MWm}^{-2}$.

For lower current operation the maximum value that avoids $\mathrm{W}$ divertor MTS melting is derived by increasing the threshold melting surface value for $15 \mathrm{MA}\left(0.6 \mathrm{MJm}^{-2}\right)$ proportionally to $\left(t_{E L M}\right)^{0.5}$, as the surface temperature rise during the ELM scales with Energy/(time $)^{0.5}, t_{E L M}$ is correlated with the ion travel time in the open field lines and thus increases for low plasma currents in ITER due to both the lower pedestal temperature and the longer SOL connection length for discharges with $q_{95}>3$ when $B$ is kept constant at $5.3 \mathrm{~T}$. The normalised ELM timescale to determine the melting threshold increase compared to that at $15 \mathrm{MA}$ is thus given by $t_{E L M} / t_{E L M, r e f}=\left(q_{95} / 3\right)\left(T_{\text {ref }} / T_{p e d}\right)^{0.5}$, where $T_{p e d}$ is the temperature at the pedestal top. In fact $q_{p k}=10 \mathrm{MWm}^{-2}$, corresponding to $T_{s}=1500^{\circ} \mathrm{C}$, is the highest stationary value of the power flux considered for the $\mathrm{W}$ divertor design and it is expected that lower divertor power flux values will be achieved routinely in ITER for lower plasma current plasmas [7]. To take into account the effect of lower divertor power fluxes between ELMs on the W divertor MTS we perform our evaluation for two values of the divertor surface temperature $T_{s}=1500^{\circ} \mathrm{C}$ and $800^{\circ} \mathrm{C}$, which correspond to values of the divertor power fluxes 
between ELMs of $q_{p k}=10 \mathrm{MWm}^{-2}$ and $5 \mathrm{MWm}^{-2}$, respectively. For the reference $15 \mathrm{MA}$ plasma conditions described above, $\varepsilon_{\max }$ is increased from $0.6 \mathrm{MJm}^{-2}$ for $T_{s}=1500^{\circ} \mathrm{C}$ to 0.8 $\mathrm{MJm}^{-2}$ when $T_{s}=800^{\circ} \mathrm{C}$.

According to the scaling, based mainly on ASDEX-U and JET experiments, the maximum ELM energy flux to the divertor parallel to the magnetic field line is proportional to the pressure at the pedestal top [4]:

$$
\varepsilon_{||}=0.084 K n_{p e d} T_{p e d} q_{95}=0.713 K n_{p e d} T_{p e d} B / I_{p}
$$

where $K=1-4.5$. The range of $K=1-4.5$ extends from the most optimistic assumptions to the most conservative assumptions regarding the ELM energy density flux within the experimental scatter and its sharing between the inner and outer divertors. The optimistic value $K=1$ corresponds to a symmetric ELM energy flux distribution between the inner and outer divertors, $\varepsilon_{\|, \text {in }}=\varepsilon_{\|, \text {out }}$ and the lowest range of the experimental scatter. This corresponds to $\varepsilon_{\|}=9.5 \mathrm{MJm}^{-2}$ for the ITER baseline $Q=10$ scenario with $I_{p} / B=15$ $\mathrm{MA} / 5.3 \mathrm{~T}, n_{\text {ped }}=810^{19} \mathrm{~m}^{-3}, T_{\text {ped }}=4.7 \mathrm{keV}$, and to $\varepsilon_{\|}=2.4 \mathrm{MJm}^{-2}$ for ITER $I_{p} / B=7.5$ $\mathrm{MA} / 2.65 \mathrm{~T}, n_{\text {ped }}=410^{19} \mathrm{~m}^{-3}, T_{\text {ped }}=2.3 \mathrm{keV}$ H-mode plasmas which will be explored already in the non-active operational phase. The value $K=4.5$ corresponds to the larger range of the experimental scatter for the outer divertor $\varepsilon_{\|, \text {out }}=3 \varepsilon_{\|, \text {out }}(K=1)=28.5 \mathrm{MJm}^{-2}$ for 15 $\mathrm{MA} / 5.3 \mathrm{~T}$ and $7.2 \mathrm{MJm}^{-2}$ for $7.5 \mathrm{MA} / 2.65 \mathrm{~T}$, and to $\varepsilon_{\|, \text {in }}=1.5 \varepsilon_{\|, \text {out }}=4.5 \varepsilon_{\|, \text {out }}(K=1)$. As mentioned above, the factor of 3 comes from the uncertainty due to the experimental data scattering and the factor of 1.5 comes from the fact that although the total ELM energy at the inner divertor is larger than at the outer one by about a factor of 2, the footprint mapped to the outer midplane is broader by about a factor of 2 in ASDEX-U [4]. This then just leaves the difference in parallel area between the inner and outer divertors which is proportional to the inverse ratio of the toroidal fields at the strike points at both divertors and this is about $B_{\text {in }} / B_{\text {out }}=1.3$ in ITER; to be conservative we take $B_{\text {in }} / B_{\text {out }}=1.5$, which is slightly higher than the factor of $\sim 1.25$ higher parallel energy fluxes at the inner divertor measured in ASDEX-U [4].

Taking into account the assumptions above, we can evaluate the limits for H-mode plasma parameters that lead to uncontrolled divertor ELM energy fluxes with acceptable transient divertor loads (i.e. no inner W divertor MTS melting) as:

$$
0.713 K \sin \alpha_{i n}\left(t_{E L M} / t_{E L M, r e f}\right)^{-0.5} n_{p e d} T_{p e d} B / I_{p}=\varepsilon_{\text {max }},
$$

where $\varepsilon_{\text {max }} \approx 0.8-0.6 \mathrm{MJm}^{-2}$ for $T_{s}=800-1500^{\circ} \mathrm{C}$.

According to the EPED1+SOLPS predictions [1] the pedestal pressure in ITER can be expressed as:

$$
p_{\text {ped }}=3.2 n_{\text {ped }} T_{\text {ped }}=2.17 B^{0.84} I_{p}
$$

From Eq. 3 the pedestal temperature is then $T_{\text {ped }}=0.85 B^{0.84} g^{-1}$ and the normalized ELM timescale $\left(t_{E L M} / t_{E L M, r e f}\right)^{0.5}=2.48 B^{0.29} g^{0.25} / I_{p}^{0.5}$. Finally, taking into account that the field line angle at the ITER inner divertor is given by $\sin \alpha_{i n} \approx 0.0197 I_{p} / B$, it is possible to derive the upper and lower ranges for the maximum plasma current in ITER for which 
uncontrolled ELMs would not cause inner W divertor MTS melting corresponding to the most optimistic and conservative assumptions regarding the divertor ELM energy fluxes that cover the experimental scatter in JET and ASDEX-Upgrade and the measured range of innerouter divertor asymmetries in ASDEX-Upgrade:

$$
\begin{aligned}
& I_{p, o p t}=(34.8-28.7) g^{0.17} B^{-0.37}, \\
& I_{p, o p t}=(12.75-10.5) g^{0.17} B^{-0.37},
\end{aligned}
$$

These upper $\left(T_{s}=800^{\circ} \mathrm{C}\right)$ and lower $\left(T_{s}=1500^{\circ} \mathrm{C}\right)$ boundaries for $I_{p}$ calculated from equations (5.1 and 5.2) are shown in figure 1 for a range of pedestal densities both with fixed magnetic fields (figure 1a,b) and with fixed safety factor (figure 1c). With the optimistic assumptions regarding ELM divertor energy deposition $(K=1)$, ELM control to avoid W inner divertor MTS melting is not required for $2.65 \mathrm{~T}$ over the whole density range up to $7.5 \mathrm{MA}$ $q_{95}=3$ while for $5.3 \mathrm{~T}$ the maximum current is limited to $10-15 \mathrm{MA}$ for $0.1<n_{\text {ped }} / n_{G W}<1$. With the conservative assumptions regarding ELM divertor energy deposition $(K=4.5)$ and $0.5<n_{\text {ped }} / n_{G W}<1$, the range where the ELM control to avoid W divertor MTS melting for $2.65 \mathrm{~T}$ is not required for $T_{s}=800^{\circ} \mathrm{C}$, is limited at 7.5 MA by $q_{95}=3$ and for $T_{s}=1500^{\circ} \mathrm{C}$ it corresponds to currents $I_{p}=6-7.4 \mathrm{MA}$. For 5.3T the values of the maximum currents that do not lead to inner $\mathrm{W}$ divertor MTS melting are lower than for $2.65 \mathrm{~T}\left(I_{p}=6-6.9 \mathrm{MA}\right.$ for $T_{s}=$ $800^{\circ} \mathrm{C}$, and $I_{p}=5-5.7 \mathrm{MA}$ for $T_{s}=1500^{\circ} \mathrm{C}$ ). The reasons for the lower values at higher B are twofold: at higher $B$ the pedestal temperature is predicted to be higher in ITER due to the pedestal being determined by the low collisionality peeling-ballooning limit (Eq. 4 ) and the area for parallel flow during the ELM decreases with increasing $B$ leading to an increase of $\varepsilon_{\|}$during the $\operatorname{ELM}$ (Eq. 2).

In the following section we estimate the requirements for ELM control in ITER from the point of view of the control of tungsten accumulation in the core plasma to ensure high performance H-mode operation.

a

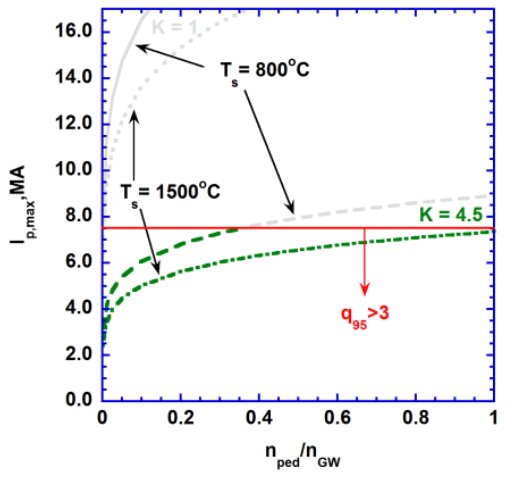

b

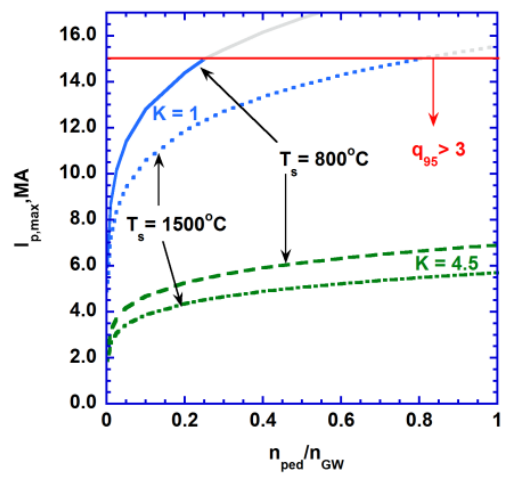

c

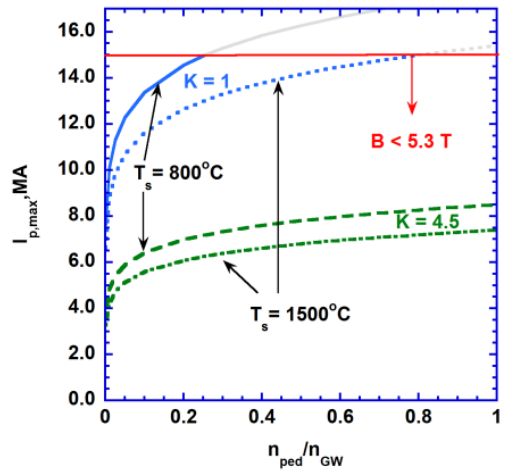

Figure 1. Maximum plasma current versus pedestal density for H-mode plasmas that avoid inner $W$ divertor MTS melting during uncontrolled ELMs, according to the optimistic and conservative assumptions for ELM energy density, for two divertor surface temperatures between ELMs, $T_{s}\left(1500^{\circ}\right.$ $C$ and $800^{\circ} \mathrm{C}$, which correspond to values of the divertor power fluxes between ELMs of $q_{p k}=10$ $\mathrm{MWm}^{-2}$ and $5 \mathrm{MWm}^{-2}$, respectively) for: $B=2.65 \mathrm{~T}(\mathrm{a}), B=5.3 \mathrm{~T}(\mathrm{~b}) ; q_{95}=3$ (c). Note that the parts of the trace in grey in (a) and (b) correspond to conditions with $q_{95}<3$, and that in (c) condition with $B$ $>5.3 \mathrm{~T}$ ). 


\section{Estimate of tungsten influx}

From experimental observations it is known [10] that the ELM energy loss and frequency are correlated with the SOL power flow as

$$
\Delta W_{E L M} f_{E L M}=\kappa P_{S O L}
$$

where we take $\kappa=0.2-0.4$ covering the range from small ELMs, $\Delta W_{E L M} / W_{\text {ped }} \ll 1$ to large ELMs. Note that in the original scaling derived in ref. [10] the ELM parameters $\Delta W_{E L M}, f_{E L M}$ are connected with the total energy, $W_{k i n}$, and the confinement time, $\tau_{E}$, by relation $\Delta W_{E L M} W_{k i n}^{-1}=\kappa\left(f_{E L M} \tau_{E}\right)^{-1}$. The energy confinement time $\tau_{E}$ was derived from the energy balance equation $\tau_{E}=W_{\text {kin }} / P_{\text {heat }}$, where $P_{\text {heat }}$ is the external heating power. Thus, the scaling can be expressed as $\Delta W_{E L M} f_{E L M}=\kappa P_{\text {heat }}$. Such expression can be applied to the case of 7.5 MA/2.65 $\mathrm{T}$ with low alpha heating and radiation. In general case the energy balance equation has the form $\tau_{E}=W_{\text {kin }} / P_{S O L}$, where $P_{S O L}=P_{\text {heat }}+P_{\alpha}-P_{\text {rad }}$. Thus, in the scaling (6) we use $P_{S O L}$ instead of $P_{\text {heat }}$. From Eq. 4 assuming $p_{e, p e d}+p_{i, p e d}=p_{\text {ped }}$ the pedestal energy of ITER plasmas is given by

$$
W_{\text {ped }}=1.5 p_{\text {ped }} V=2.68 B^{0.84} I_{p}
$$

for the typical plasma volume $V=820 \mathrm{~m}^{3}$. For ITER H-mode plasmas at $15 \mathrm{MA} / 5.3 \mathrm{~T} W_{\text {ped }}=$ $163 \mathrm{MJ}$, while for H-mode plasmas at 7.5MA/2.65T $W_{\text {ped }}=45.5 \mathrm{MJ}$. The ELM energy loss of uncontrolled ELMs in ITER can be evaluated from the empirical relation [11], $\triangle W_{E L M} W_{\text {ped }}^{-1}=0.064\left(v_{p}^{*}\right)^{-1 / 3} \approx 0.118 B^{0.224} g^{-1} \sim 0.15-0.2 \quad, \quad$ where $v_{p}^{*}=8.6910^{-4} \pi R q_{95} n_{\text {ped }} T_{p e d}^{-2} \approx 0.16 g^{3} B^{-0.68}$ is the pedestal collisionality expressed using eq. (4). Thus, from equation (7), the uncontrolled ELM energy loss in ITER is given by

$$
\Delta W_{E L M} \approx 0.315 I_{p} B^{1.064} g^{-1} .
$$

From Eqs. (5.2) and (8) we thus obtain for the most conservative assumptions the largest uncontrolled ELMs that avoid inner W divertor MTS melting $\Delta W_{E L M, \max }$ (figure 2). The uncontrolled ELM frequency can be evaluated from Eqs. (6) and (8) and corresponds to $f_{E L M} \sim 0.03-0.06 g P_{S O L}$ for $7.5 \mathrm{MA} / 2.65 \mathrm{~T}$ and $f_{E L M} \sim 0.007-0.014 g P_{S O L}$ for $15 \mathrm{MA} / 5.3 \mathrm{~T}$.

In order to determine if the uncontrolled ELM frequency is sufficient to provide $\mathrm{W}$ accumulation control in ITER or not and, if not, which ELM frequency would be required, we have used the results of previous simulations of $\mathrm{W}$ divertor production and transport $[3,8]$ and evaluated the time-averaged $\mathrm{W}$ influx into the confined plasma for a range of ELM frequencies, edge power flows, $P_{S O L}$, and assumptions regarding $\mathrm{W}$ re-deposition during the ELM. We have also analyzed the impact on H-mode operation of considering discrete influx pulses of tungsten sputtered by ELMs that reach the plasma core instead of the time-averaged influx values.

To estimate the dynamics of $\mathrm{W}$ accumulation in ITER we have evaluated analytical expressions that reproduce the characteristics of the tungsten influx to the plasma core as a function of the ELM energy loss from results of combined simulations of ITER scenarios by ASTRA, STRAHL, and SOLPS codes described in $[3,8]$. In $[3,8]$ it was found that the time 
that it takes to the $\mathrm{W}$ to reach the core plasma after the ELM, $\Delta t_{d, W}$, and the duration of the tungsten core plasma influx due to the ELM, $\Delta t_{p, W}$, depend on the ELM energy loss as:

$$
\begin{aligned}
& 1000 \Delta t_{d, W}=0.3+1.8 \exp \left(-3.7 \Delta W_{E L M}\right), \\
& 1000 \Delta t_{p, W}=1.3+7 \exp \left(-2.2 \Delta W_{E L M}\right) .
\end{aligned}
$$

For the most conservative case in which no prompt re-deposition of the sputtered tungsten during the ELM is considered, the number of particles entering the core plasma after the ELM has been evaluated from the results in $[3,8]$ and found to be well described by the fit:

$$
\Delta N_{W}=2.810^{18}\left(1-\exp \left(-\Delta W_{E L M}^{1.6}\right)\right) \text {. }
$$

The corresponding prompt and time-averaged tungsten influxes by ELMs are thus given by:

$$
\begin{aligned}
& S_{W}=\Delta N_{W} \Delta t_{p, W}^{-1}=2.810^{21}\left(1-\exp \left(-\Delta W_{E L M}^{1.6}\right)\right)\left(1.3+7 \exp \left(-2.2 \Delta W_{E L M}\right)\right)^{-1}, \\
& <S_{W}>=\Delta N_{W} f_{E L M}=2.810^{18} \kappa P_{S O L}\left(1-\exp \left(-\Delta W_{E L M}^{1.6}\right)\right) \Delta W_{E L M}^{-1}
\end{aligned}
$$

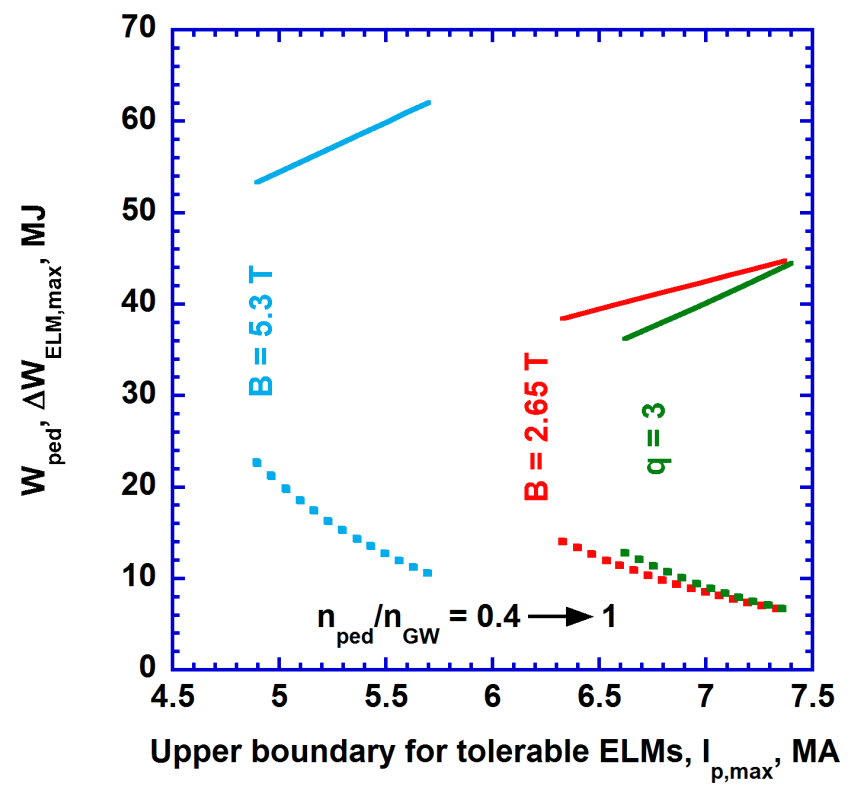

Figure 2. Pedestal energy, $W_{\text {ped }}$ (bold), and uncontrolled ELM energy loss, $\triangle W_{E L M}$, (dots) for ELMS which do not lead to inner $W$ divertor MTS melting with conservative assumptions for the ELM energy flux $(K=4.5)$ for $T_{s} \sim 1500^{\circ} \mathrm{C}$, for density scans both at $B=$ const (Fig. 1a,b) and at $q=3$ (Fig. 1c). The direction of the density increase is shown by arrow. where Eq. (6) has been used to relate $f_{E L M}$ and $P_{S O L}$. It is important to note that considering $\mathrm{W}$ redeposition during the ELMs can reduce the values of the $\mathrm{W}$ influxes given by Eqs. (11.1) and (11.2) by more than a factor of 100 [13].

The prompt and time-averaged tungsten influxes are shown in figure 3 . The solid lines correspond to $P_{S O L}=50 \mathrm{MW}$ and the dashed lines to $P_{S O L}=100 \mathrm{MW}$ both with $\kappa=0.2$. Note that the timeaveraged influx for $\mathrm{P}_{\mathrm{SOL}}=100 \mathrm{MW}$ has a maximum at a frequency $f_{E L M} \sim 30 \mathrm{~Hz}$, which is required to achieve controlled ELMs with $\Delta W_{E L M}=0.6 \mathrm{MJ}$ by pellet pacing [1]. On the other hand the prompt $\mathrm{W}$ influx into the plasma drops significantly with increasing ELM frequency beyond $5-10 \mathrm{~Hz}$ so that $S_{W}(30 \mathrm{~Hz}) / S_{W, \max } \sim 0.25$ for baseline 15 MA, $100 \mathrm{MW}$ case (figure 3b).

The different behaviour of the time-averaged $\mathrm{W}$ influx and of the prompt influx due to ELMs versus ELM frequency indicates that considering the discrete nature of the ELM on the W influx and that of the triggering of the ELMs by pellet can lead to different results from those obtained previously regarding $\mathrm{W}$ accumulation control with continuous models for ELMs and pellets [1]. The difference in results with these two modelling approaches is discussed in the next section using the tungsten influx discussed in this section. 


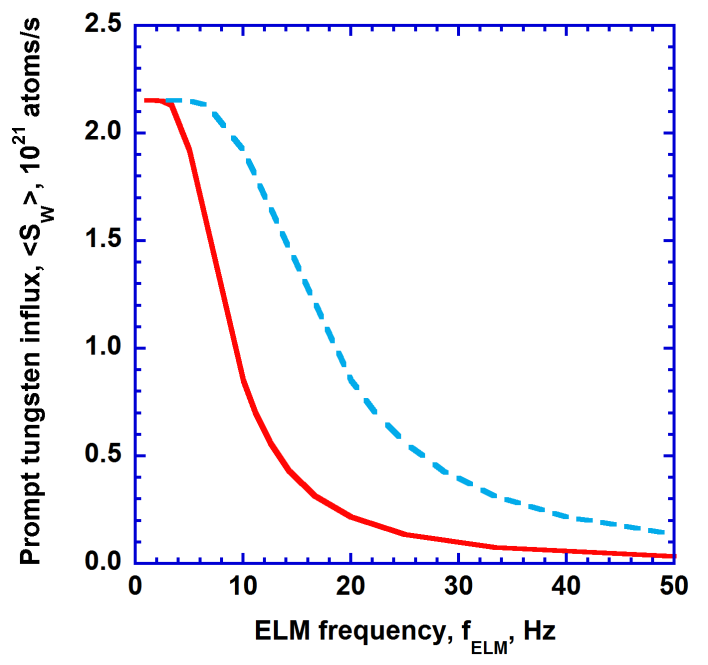

a

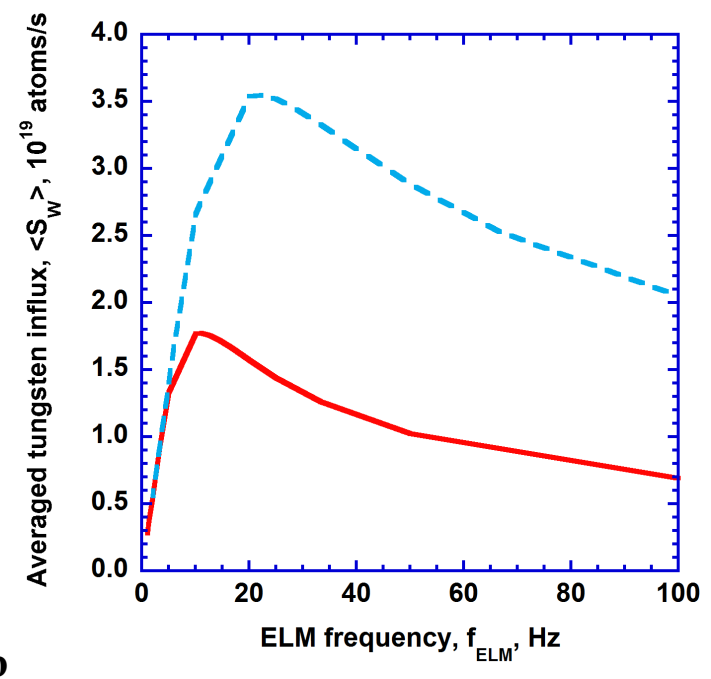

Figure 3. Influx of tungsten sputtered by ELMs to the core without prompt $W$-re-deposition for $P_{S O L}=100 \mathrm{MW}$ (dashed line) and $P_{S O L}=50 \mathrm{MW}$ (solid line) with $\kappa=0.2:$ (a) - prompt influx (see equation (11.1)); (b)- time averaged flux (see equation (11.2)).

\section{Impact of the discrete pellet and ELM modelling on plasma performance}

In our previous simulations [1] with continuous ELM and pellet models we applied 1.5D transport simulations of the core plasma heat, particle and poloidal flux evolution with boundary conditions and edge fuelling consistent with SOLPS results [14] for ITER which consistently takes into account ELM pacing, pellet fuelling, pumping and the divertor detachment control [1]. The transport coefficients in the pedestal were chosen to provide the inter-ELM pedestal height consistent with the EPED1+SOLPS predictions for ITER [5], neon injection and gas puffing was applied for the control of the power loads, high field side pellet injection provided core plasma fuelling and low field side pellet injection was applied for ELM control. Core modelling was performed by 1.5D transport analysis on the basis of the Automated System for Transport Analysis (ASTRA) [15] with the ZIMPUR code for impurity transport [16] using the results of ASTRA-STRAHL and SOLPS simulations [3] for the time-averaged tungsten influxes (Eq. 11.2). For the simulation of impurity ions we assume boundary conditions which provide quasi-neutrality, $G_{i}=n_{i} G_{e} / \sum Z_{i} n_{i}$, where $n_{i}, Z_{i}, G_{i}$ are the ion density, charge state and out-flux, $G_{e}$ is the outflux of the electrons.

In our present studies we extend the considerations in [1] by including the modelling of discrete ELMs [3] and of discrete pellets [12]. To determine the effect of discrete pellets, we have modelled the ablation and drift of pellet injected particles from LFS and HFS in ITER. According to model predictions [12], the drift of the ablated pellet particles in the direction of the LFS is larger for larger pellets and even the smallest LFS pellets, $V_{p e l}=33 \mathrm{~mm}^{3}$, is sufficient for the triggering of ELMs in $15 \mathrm{MA} / 5.3 \mathrm{~T}$. In addition, the relocation of the ablated pellet toward the LFS is so large that the residual core plasma fuelling of LFS pellets injected for ELM control is negligible (see figure 4). Therefore, the modelling with the continuous pellet model in [1] that considered the possibility of independent control of ELM triggering by LFS pellets and plasma fuelling by HFS injected pellets appears to be valid for modelling with discrete pellets. Note that particles expelled by ELMs practically do not penetrate back to the core due to opaque SOL [1]. The energy loss in small controlled ELMs is assumed to be fully convective [1]. 
To model the effect of discrete ELMs on the plasma we consider both diffusive and convective ELM models [3] for the particle losses by ELMs. The diffusive ELM is modelled by increased particle diffusivity, resulting in a flattening of fuel and impurity profiles in the ELM-affected area, $\Delta_{E L M}$, while the convective ELM ejects plasma particles by a net outward convective velocity over the same area. The ELM frequency, $f_{E L M}$, is considered as a variable parameter controlled by HFS-fuelling and LFS-pacing pellets, $f_{p e l}=f_{E L M}=f_{H F S}+f_{L F S}$, assuming that all injected pellets induce ELMs connected with ELM size, $\Delta W_{E L M}$, by Eq. (6). Note that the post-pellet reversed density gradients after fuelling HFS pellets affects postELM plasma profiles and lead to an increased $\mathrm{W}$ outflux in the ELM-affected area due to neoclassical transport effects [20]. To simulate the ELMs the relevant transport coefficients were increased for $\Delta t_{E L M} \sim 1 \mathrm{~ms}$ to provide the energy loss, $\Delta W_{E L M}$, calculated from Eq. (6) by increasing particle diffusivity for diffusive ELM model or outward particle pinch for convective model for main species and impurities. For $\mathrm{W}$ transport inter-ELM neoclassical transport in the pedestal is considered. During the ELM we assume that in the ELM affected area the same transport assumptions and transport coefficient increases used to simulate the ELM particle expulsion for the main ions also apply to all impurities including W.
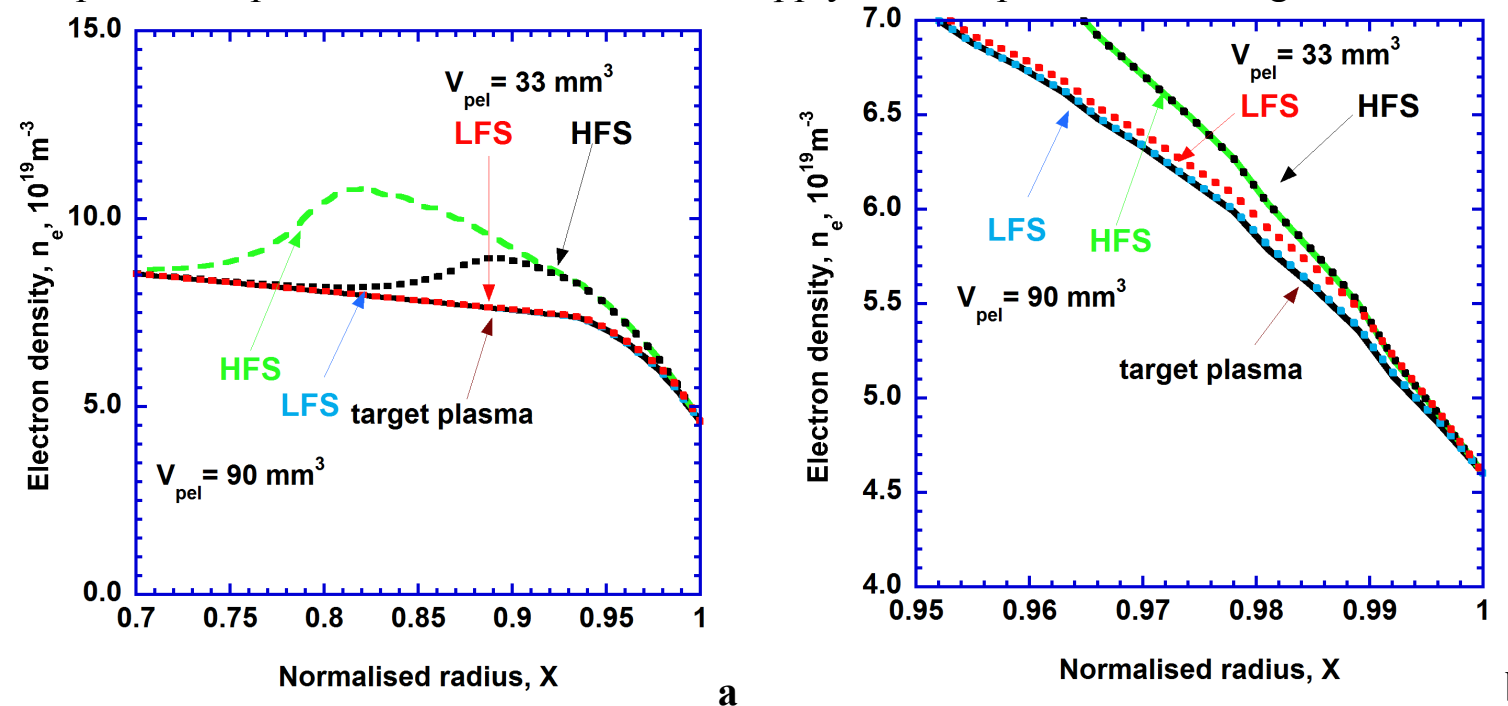

Figure 4. Comparison of particle deposition after the drift towards the low field direction for LFS and $H F S$ injection of DT pellet of different sizes with pellet speed $v=300 \mathrm{~m} / \mathrm{s}$ and geometry described in [17] for 15 MA/5.3T Q=10 plasmas (assuming that an ELM is not triggered during the material relocation phase): (a)-whole radial profile; (b)-zoomed to the pedestal region.

a

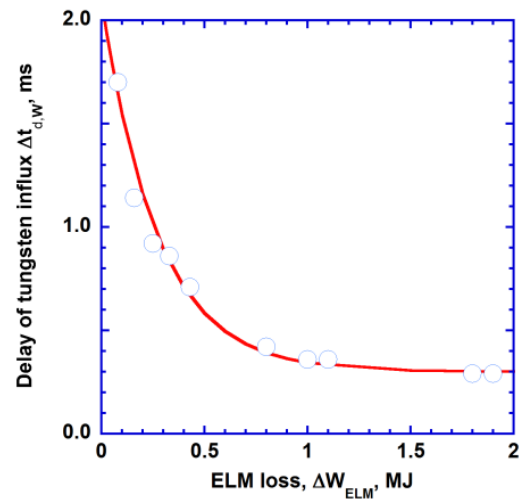

b

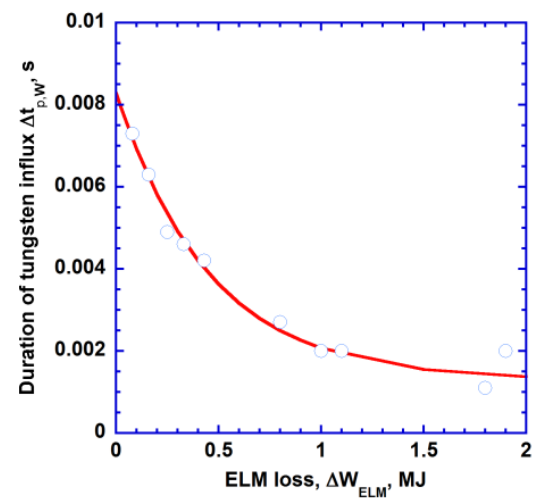

c

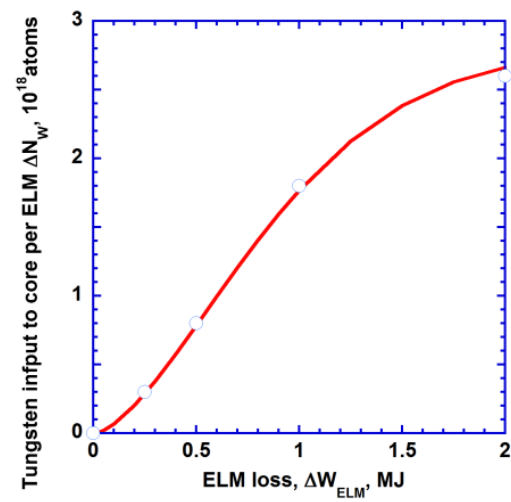


Figure 5. Characteristics of the post-ELM tungsten influx pulse which reaches plasma w/o prompt redeposition: (a) - delay of $W$ influx; (b) - influx pulse duration; (c) - W particle input per ELM. Fitted data are shown by circles, analytic fitting by formulas (9.1), (9.2) and (10) is shown by solid lines.

With the discrete ELM modelling studies we address two key issues: what is the magnitude of the peak $\mathrm{W}$ radiation in the plasma following the ELM, which could cause $\mathrm{H}$ to L-mode transitions due to the transiently reduced edge power flux as observed in some experiments [18]; and the accumulation of $\mathrm{W}$ in the core plasma on long timescales. The characteristics of the ELM-caused $\mathrm{W}$ influx pulses, which reach the plasma core, are shown in figure 5 for the case without prompt $\mathrm{W}$ re-deposition. We assume absence of the hysteresis for the $\mathrm{H}-\mathrm{L}$ transition.

The magnitude of the $\mathrm{W}$ pulse influx and its duration saturate at $\Delta W_{E L M} \sim 2 \mathrm{MJ}$. For larger ELM energy losses the particle losses caused by the ELM remain constant and only conductive losses (associated with the decrease of the pedestal temperature) increase so that the total sputtered W influx does not increase beyond 2 MJ for ITER plasmas [19]. Thus, to address the instantaneous effects of single ELMs it is sufficient to consider ELMs with up to that energy loss. The $\mathrm{W}$ influx-pulse that reaches the core plasma from the divertor increases strongly the level of plasma radiation in the confined plasma. When this increase is sufficiently large, it can promptly reduce the power to the SOL in the post-ELM phase to values below the L-H threshold [20] leading to an $\mathrm{H}$ to $\mathrm{L}$ transition possibly followed by a disruption.

To characterize the effect of W accumulation over long timescales it is useful to introduce the tungsten accumulation factor, $F_{W, a}=f_{E L M} \times t_{W}$, where the full $\mathrm{W}$ recovery time, $t_{W}$, includes the post-ELM W influx-pulse delay and duration times, $\Delta t_{d, W}, \Delta t_{p, W}$ (see equation (9)), as well as the recovery time, $t_{W, \text { rec }}$, i.e. time required to remove the $\mathrm{W}$, delivered by the $\mathrm{W}$ influx-pulse, from the plasma core: $t_{W}$ $=\Delta t_{d, W}+\Delta t_{p, W}+\mathrm{t}_{W, r e c}$. The recovery time, $t_{W, \text { rec }}$ (figure 6) is calculated on the basis of time-dependent $1.5 \mathrm{D}$ transport modelling described in [1]. Note that an accumulation factor greater than unity, $F_{W, a} \geq 1$ means that tungsten delivered by the $\mathrm{W}$ influxpulse cannot be fully removed between ELMs, which causes tungsten accumulation, a gradual increase of core radiation, reduction of the power flux to the SOL

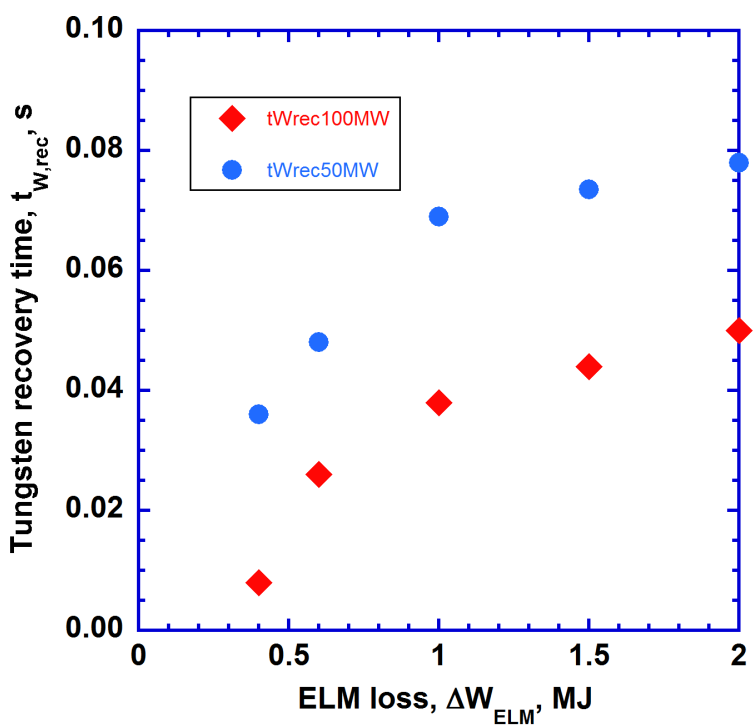

Figure 6. Time of post-ELM recovery of plasma purity $t_{W, \text { rec }}$ for $H$-mode plasmas in ITER with $I_{p} / B$ $=15 \mathrm{MA} / 5.3 \mathrm{~T}$ and $P_{S O L}=100 \mathrm{MW}$, and with $I_{p} / B=$ $7.5 \mathrm{MA} / 2.65 \mathrm{~T}$ and $P_{S O L}=50 \mathrm{MW}$ w/o prompt tungsten re-deposition for diffusive ELMs, $f_{E L M}=0.2 P_{\text {sol }} / \Delta W_{E L M}$ which goes under the L-H

threshold and thus a follow-up transition to L-mode. It should be noted here that for diffusive ELMs the dynamics of $\mathrm{W}$ accumulation in ITER is opposite to that usual in present experiments. Due to neoclassical transport effects in the ITER pedestal W is well screened so that the net $\mathrm{W}$ outflux is outwards between the ELMs and the $\mathrm{W}$ density profiles are hollow in the pedestal region [19]. With the ELM diffusive model assumptions this leads to a net W 
influx being produced at each ELM and thus to the possible increase of the $\mathrm{W}$ concentration in the plasma following the ELM. The results of simulations of post-W influx-pulse plasma parameters are presented in figure 7.

According to these simulations, the influxes of $\mathrm{W}$ which reach the core when no prompt redeposition is assumed can increase prompt radiation after the ELM to the level sufficient to trigger the H-L transition already for very moderate ELM energy losses $\Delta W_{E L M} \geq 1.2 \mathrm{MJ}$ for both $15 \mathrm{MA} / 5.3 \mathrm{~T}$ and $7.5 \mathrm{MA} / 2.65 \mathrm{~T}$. On the other hand, regarding $\mathrm{W}$ accumulation for the range of frequencies required to control the ELM power loads at $15 \mathrm{MA} / 5.3 \mathrm{~T}$ and $P_{S O L}=100$ MW by pellet pacing up to $f_{\text {pel }}=50 \mathrm{~Hz}$ (and $\Delta W_{E L M}=0.4 \mathrm{MJ}$ for $\kappa=0.2$ ), W accumulation does not occur, although the conditions for $f_{p e l}=32 \mathrm{~Hz}$ (and $\Delta W_{E L M}=0.63 \mathrm{MJ}$ ) are marginal. For at $7.5 \mathrm{MA} / 2.65 \mathrm{~T}$ and $P_{S O L}=50 \mathrm{MW}$ gradual accumulation of $\mathrm{W}$ can occur at lower frequencies when $f_{p e l} \geq 25 \mathrm{~Hz}$ and (and $\Delta W_{E L M} \leq 0.4 \mathrm{MJ}$ ).
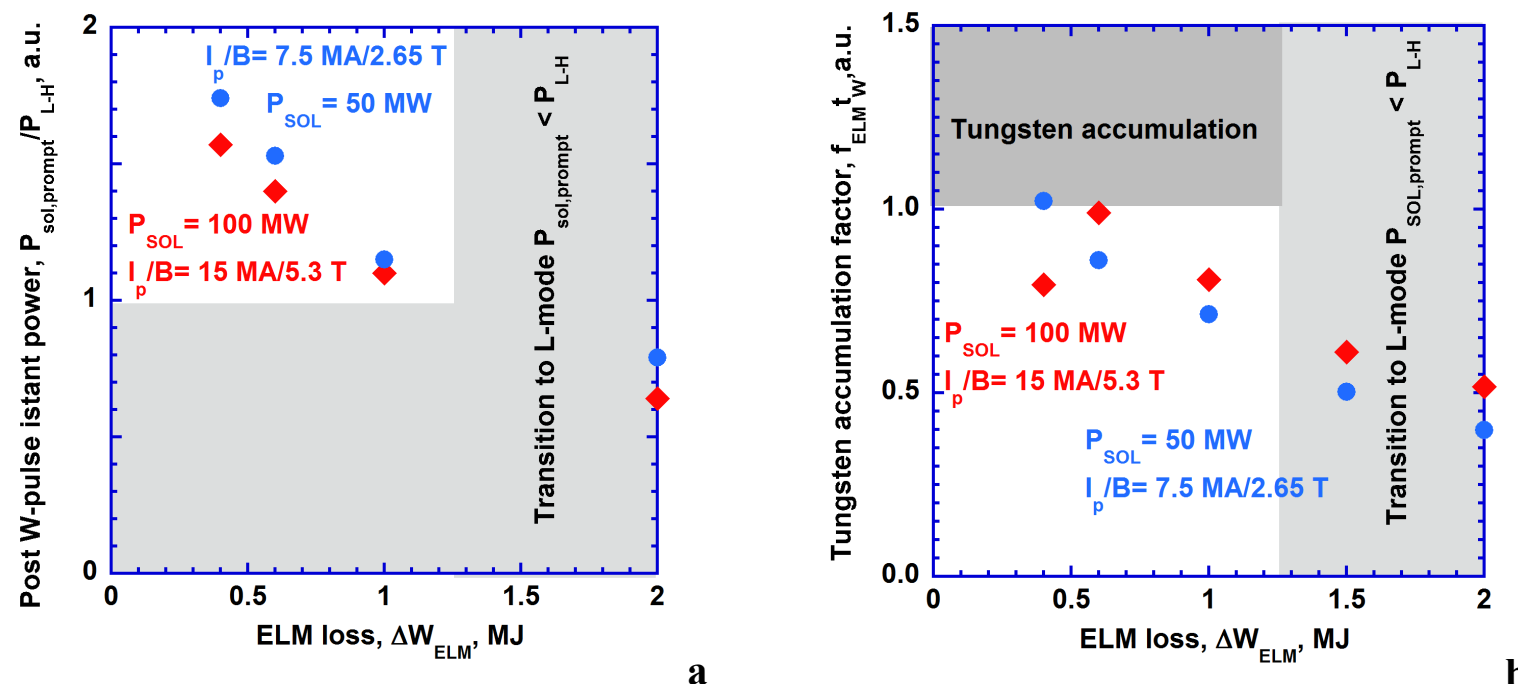

Figure 7. H-mode operating space for $I_{p} / B=15 M A / 5.3 T$ with $P_{S O L}=100 \mathrm{MW}$, and for $I_{p} / B=$ 7.5MA/2.65 T with $P_{S O L}=50 \mathrm{MW}$ w/o tungsten re-deposition with $f_{E L M}=0.2 P_{S O L} / \Delta W_{E L M}$. The ratio of power to SOL, $P_{S O L \text {,prompt }}$ dropped due to the post $W$-influx, to the L-H power threshold is shown at figure (a). Condition for $H$-mode operation $w / o$ the hysteresis $P_{S O L \text {,prompt }} / P_{L-H}>1$ restricts the ELM size at the level $\Delta W_{E L M} \leq 1.2$ both for convective and diffusive ELM models. Restrictions for diffusive ELM model caused by tungsten accumulation factor, $F_{W, a}=f_{E L M} \times t_{W, r e c} / \Delta W_{E L M} \leq 1$ are shown in (b) by dark grey. For convective model the ELM size is restricted only by $\Delta W_{E L M} \leq 1.2$, caused by prompt $H-L$ transition due to high core radiation. 
It is important to note that the uncontrolled ELMs that do not produce inner $\mathrm{W}$ divertor MTS melting are much larger (see figure 2 a) than the $1.2 \mathrm{MJ}$ that causes prompt transition to L-mode for $15 \mathrm{MA} / 5.3 \mathrm{~T}$ and 7.5 MA/2.65T plasmas when no $\mathrm{W}$ re-deposition is considered and thus are not compatible with sustained H-mode operation for these assumptions. If sizeable $\mathrm{W}$ prompt redeposition during the ELM takes place, as proposed in [13], the issues regarding both the transient increase of plasma radiation following the ELM as well as of long timescale $\mathrm{W}$ accumulation with a diffusive ELM model disappear. Re-deposition during the ELMs is predicted to reduce the $\mathrm{W}$ influxes from the divertor by a factor of typically $\sim 10^{2}-10^{3}$ times (and for full prompt re-deposition by a factor of $10^{4}-10^{5}$ ) compared to those without prompt re-deposition [13] and this decreases

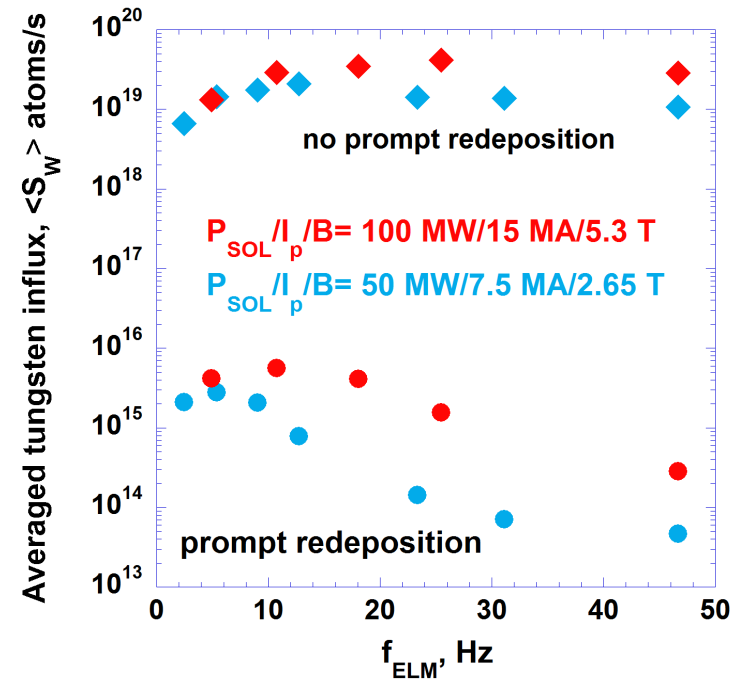

Figure 8. Time-averaged tungsten flux to the core, $S_{W}$, induced by ELMS with frequency $f_{E L M}=0.2 P_{\text {sol }} \Delta W_{E L M}$ for $15 M A / 5.3 T$ with $P_{S O L}$ $=100 \mathrm{MW}$ and $7.5 \mathrm{MA} / 2.65 \mathrm{~T}$ with $P_{S O L}=50$ $M W$ with and without prompt $W$ redeposition proportionally the prompt $\mathrm{W}$

radiation during the ELM as well as the $\mathrm{W}$ influx in the plasma following the ELM and the W time-averaged influx (see figure 8, produced from modelling results in [8]). It should also be noted that the issue of long timescale $\mathrm{W}$ accumulation with increasing ELM frequency is a specific feature of the diffusive ELM model. When ELM particle expulsion is modelled with a convective model, long term $\mathrm{W}$ accumulation decreases with increasing ELM frequency for all ITER plasma conditions modelled. For full tungsten prompt re-deposition the ELM frequency is not limited by core contamination by tungsten.

\section{Discussion and conclusions}

We have used EPED1+SOLPS predictions for pedestal height and the empirical dependence of the ELM parallel energy density on pedestal parameters to derive the expected parallel energy fluxes during ELMs in ITER. This has allowed the derivation of the boundaries of the H-mode operating space in terms of $I_{p}$ and $B$, where uncontrolled ELMs will not cause melting of the $\mathrm{W}$ divertor monoblock top surface.

The analysis carried out by the application of then ELM energy density scaling in [4] together with the pedestal plasma pressure derived from EPED1+SOLPS predictions [5] indicates that ELM control to avoid melting of the inner $\mathrm{W}$ divertor monoblock top surface would not be required in ITER for $2.65 \mathrm{~T}$ operation with $q_{95} \geq 3$ and $n_{\text {ped }} / n_{G W} \geq 0.3$ for Hmodes in which the divertor power flux between ELMs does not exceed $5 \mathrm{MWm}^{-2}$. If the power flux reaches $10 \mathrm{MWm}^{-2}$ the maximum plasma current would be in the range of 6.0-7.5 MA for $0.5<n_{\text {ped }} / n_{G W}<1.0$ for the most conservative assumptions regarding the ELM energy density and in/out divertor asymmetry. For $5.3 \mathrm{~T}$ operation the maximum current that avoids inner W divertor MTS melting without ELM control are somewhat lower than those at $2.65 \mathrm{~T}$ due to the almost linear scaling of the pedestal plasma pressure with toroidal field in the peeling-limited pedestal plasmas of ITER ( $p_{p e d} \sim I_{p} \times B$ in equation (4)). For the most optimistic assumptions regarding ELM energy flux densities $(K=1)$ and divertor power fluxes between ELMs $\left(q_{p k}=5 \mathrm{MW} / \mathrm{m}^{2}\right)$, inner W divertor MTS melting does not occur for 
plasma currents up to $15 \mathrm{MA}$ for H-modes with $0.5<n_{\text {ped }} / n_{G W}<1.0$. The actual maximum plasma current values of the present estimates are typically somewhat higher than previous studies for $q_{95}=3$ assuming the same wetted area during ELMs and between ELMs [2]. This is the due to: a) the new findings in [4] relating the ELM energy flux during ELMs to the pedestal pressure, which results in an almost linear increase of the ELM wetted area with $\Delta W_{E L M}$ for fixed pedestal pressure and b) different assumptions regarding the inner to outer ELM energy deposition asymmetries. In [2] it was assumed that the ELM energy flux at the inner divertor was a factor of 2 larger than at the outer one, which is now questionable on the basis of the new results from ASDEX-Upgrade in [4] showing a more symmetric distribution (a factor of $\sim 1.25$ in experiments, 1.5 assumed for the ITER modelling presented here).

It should be noted that the corresponding ELM energy losses for these uncontrolled ELMs that avoid inner W divertor MTS melting are very large (typically $\sim 10 \mathrm{MJ}$ ) and their frequency is low (typically $f_{E L M} \sim 1-2 \mathrm{~Hz}$ ). Although these large ELMs may avoid W divertor MTS melting it is unlikely that sustained ITER H-mode operation will be possible in these conditions both because of the resulting energy fluxes on the first wall associated with the broadened ELM energy deposition and the large $\mathrm{W}$ influxes into the confined plasma caused by physical sputtering during the ELMs which severely impact H-mode operation.

Previous simulations performed taking into account time-averaged $\mathrm{W}$ influxes caused by ELMs [1] showed that neoclassical screening of the $\mathrm{W}$ in the plasma pedestal is sufficient to keep H-mode operation even for the largest time-averaged $\mathrm{W}$ influxes without prompt redeposition both for $15 \mathrm{MA} / 5.3 \mathrm{~T}$ with $P_{S O L}=100 \mathrm{MW}$ and for $7.5 \mathrm{MA} / 2.65 \mathrm{~T}$ with $P_{S O L}=50$ MW because the separatrix densities required for semi-detached divertor operation are sufficiently high to provide efficient neoclassical screening of tungsten [3,20]. However, the full 1.5D modelling of plasma evolution with discrete ELMs and $\mathrm{W}$ influx pulses described in this paper shows that limitations on the ELM size appear when significant influxes of $\mathrm{W}$ sputtered by ELMs reach the core plasma, although the precise value of the ELM size limit depends on the assumptions regarding $\mathrm{W}$ prompt re-deposition during the ELMs. Without W re-deposition, ELMs of rather moderate size $\Delta W_{E L M}>1.2 \mathrm{MJ}$ can produce $\mathrm{W}$ influx-pulses that increase core plasma radiation to a level sufficient to trigger back H-L transition for 15 $\mathrm{MA} / 5.3 \mathrm{~T}$ and $7.5 \mathrm{MA} / 2.65 \mathrm{~T}$, possibly followed by a disruption, in agreement with findings in [3]. For diffusive ELMs with $\Delta W_{E L M} \leq 1.2 \mathrm{MJ}$ another limitation to $\mathrm{H}$-mode operation can come from tungsten accumulation on long timescales in ITER. In this case the dominant transport process during the ELM strongly affects tungsten accumulation for ITER predictions because tungsten is screened by neoclassical transport in the pedestal of ITER plasmas between ELMs and thus W expulsion occurs between ELMs. For ELMs modelled with a convective model the additional tungsten coming to the core after an ELM is efficiently pushed out by the following ELM. Thus, both the inter-ELM W expulsion and the convective expulsion are aligned in ITER. Indeed with the convective ELM modelling assumptions the neoclassical screening remains always effective, in agreement with previous studies $[3,7]$. On the contrary, for ELMs modelled with a diffusive model, the ELM flattens the tungsten profile producing a deeper penetration of tungsten after each ELM in the case of ITER plasma due to the effective W screening between ELMs. Thus, for diffusive ELMs tungsten can accumulate in the plasma core when the tungsten recovery time, $t_{W}$, is larger than the time between ELMs, i.e. $f_{E L M} \times t_{W, \text { rec }}=\kappa P_{\text {sol }} t_{W, \text { rec }} / \Delta W_{E L M} \geq 1$. Our modelling with $\kappa=0.2$ predicts no tungsten accumulation for ELM controlled frequencies up to $50 \mathrm{~Hz}$ which are achieved by pellet pacing for $15 \mathrm{MA} / 5.3 \mathrm{~T}$ plasmas with $P_{S O L}=100 \mathrm{MW}$, although the conditions for $f_{\text {pel }}=32 \mathrm{~Hz}\left(\Delta \mathrm{W}_{\mathrm{ELM}}=0.6 \mathrm{MJ}\right)$ are marginal. For $15 \mathrm{MA} / 5.3 \mathrm{~T}$ plasmas with 
$P_{S O L}=100 \mathrm{MW}$ assuming $\kappa=0.4$, ELMs leading to $\Delta W_{E L M}=1 \mathrm{MJ}$ with $f_{E L M}=40 \mathrm{~Hz}$, have a $\mathrm{W}$ accumulation factor of $\sim 1$.7. In this case tungsten gradual accumulation occurs and this causes a reduction of the SOL power flow under the L-H threshold, which is in agreement with the simulations in [7]. It should be noted that the limit on pellet-controlled ELM frequency associated with the gradual tungsten accumulation depends on the power to SOL and plasma parameters. In particular, for $7.5 \mathrm{MA} / 2.65 \mathrm{~T}$ plasmas with $P_{S O L}=50 \mathrm{MW}$, gradual accumulation of tungsten starts at lower pellet-controlled ELM frequencies, $f_{\text {pel }} \geq 25 \mathrm{~Hz}$.

The minimal pellet frequency corresponds to the maximal ELM size, $\Delta W_{E L M}=1.2 \mathrm{MJ}$. For $7.5 \mathrm{MA} / 2.65 \mathrm{~T}$ plasmas with $P_{S O L}=50 \mathrm{MW}$ the minimal frequency is about $8.5 \mathrm{~Hz}$, for 15 MA/5.3 T plasmas with $P_{S O L}=100 \mathrm{MW}$ it is about $17 \mathrm{~Hz}$.

In general, in agreement with findings in $[2,19]$ both instant L-H transitions and $\mathrm{W}$ accumulation with a diffusive ELM model occur for much smaller ELM energy losses than those required to avoid $\mathrm{W}$ divertor MTS melting, when no prompt re-deposition of $\mathrm{W}$ during ELMs is considered. To refine the ITER estimates described in this paper it is necessary to have a validated model for $\mathrm{W}$ re-deposition during ELMs with, as far as possible, ITER-like ELM conditions (i.e. $T_{p e d}>1 \mathrm{keV}$ ). The need for such validated model is even more important for the description of particle and impurity expulsion during the ELMs. In this case the essential feature of the experiments to validate such model is that these are performed in conditions in which impurity screening dominates impurity dynamics between ELMs in the pedestal. Without this it is not possible to distinguish the differences in impurity expulsion between diffusive and convective models for the ELMs. In addition, from the modelling side, to have a more accurate assessment of the evaluation for ITER direct ASTRA-SOLPS coupling is required, following the lines of JETTO-EDGE2D coupling, which could be the subject of future work for further investigations for ITER.

Disclaimer: ITER is the Nuclear Facility INB no. 174. The views and opinions expressed herein do not necessarily reflect those of the ITER Organization. This work has been carried out within the framework of the EUROfusion Consortium and has received funding from the Euratom research and training programme 2014-2018 under grant agreement No 633053. The views and opinions expressed herein do not necessarily reflect those of the European Commission.

\section{Acknowledgements}

We are thankful to Dr. R.A. Pitts and Dr. A.S. Kukushkin for useful advice on our ITER simulations and constructive comments to the paper, and to Dr. D.J. Campbell for support of our studies.

\section{References}

[1] A.R. Polevoi, et al., Nucl. Fusion, 57 (2017) 022014

[2] A. Loarte, et.al, Nucl. Fusion, 54 (2014) 033007

[3] R. Dux, et. al, "Influence of a Tungsten Divertor on the Performance of ITER H-Mode Plasmas", TH/P3-29, 25th IAEA Fusion Energy Conference, 13-18 October, 2014, Saint Petersburg, Russian Federation.

[4] T. Eich, et al, Proc. $22^{\text {nd }}$ International Conference on Plasma Surface Interactions in Controlled Fusion Devices (PSI), 2016, Rome, Italy.

[5] A.R. Polevoi et. al, Nucl. Fusion, 55 (2015) 063019

[6] J. Gunn, et al., Nucl. Fusion, 57 (2017) 046025 
[7] R.A. Pitts, et al., Nuclear Materials and Energy, 2017, article in press

[8] D.P. Coster, et al, J. Nucl. Mater 463 (2015) 620

[9] T. Eich et al. , J. Nucl. Mater., 415 (2011) S856-S859

[10] A. Hermann, Plasma Phys. Contr. Fusion 44 (2002) 883

[11] A. Loarte et al, Plasma Phys. Control. Fusion 44 (2002) 1815

[12] A.R. Polevoi and M. Shimada, Plasma Phys. Control. Fusion 43 (2001) 1525

[13] A.V. Chankin, et al, Plasma Phys. Control. Fusion 56 (2014) 025003

[14] H.D. Pacher, et al, J. Nucl. Mater. 463 (2015) 591-595

[15] G.V. Pereverzev and P.N. Yushmanov, "ASTRA Automated System for TRansport Analysis", IPP-Report IPP 5/98 (2002).

[16] V.M. Leonov, V.E. Zhogolev, Plasma Phys. Control. Fusion, 47 (2005) 903

[17] S. Maruyama et al," ITER Fuelling and Glow Discharge Cleaning System Overview", ITR/P1-28, $23^{\text {rd }}$ IAEA Fusion Energy Conference Proceedings 10-16 October 2010, Daejon, Republic of Korea

[18] P. C. de Vries, et al, Phys. Of Plasmas , 21 (2014) 056101

[19] R. Dux, et al, Plasma Phys. Control. Fusion, 56 (2014) 124003

[20] Y.R. Martin, et al, Journal of Physics: Conference Series 123 (2008) 012033 